\title{
Symplectic Structure of Isospin Particles in Yang-Mills Fields
}

\author{
Phillial $\mathrm{Oh}^{*}$ \\ Departruent of Physics \\ minge moT \\ University of Florida
}

Gainesville, FL 32611

\begin{abstract}
Using Dirac's constraint analysis, we explore the Hamiltonian formalism of isospin particles in external Yang-Mills fields without kinetic and potential energy term. We consider an example of isospin particle in ' $t$ Hooft-Polyakov magnetic monopole field and discuss possible quantization condition of magnetic charge in terms of geometric quantization.
\end{abstract}

* Permanent address: Department of Physics, Sung Kyun Kwan University, Suwon 440-746, Korea

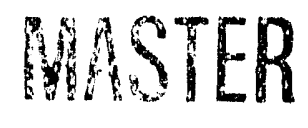

OISTRIBUTION OF THIS DOCUMENT IS UNLIMITED 
Chern-Simons quantum mechanics[1] (CSQM) and its supersymmetric generalization [2] have received much attention recently in relation with topological field theory[3]. Although dynamically trivial, this system has many interesting properties[4] and serves as a toy model for topological field theory.

In ref. [1], Hamiltonian formalism of CSQM was investigated in detail using Dirac's constraint analysis[5]. The symplectic structure on the reduced phase space was given by the field strength two-form of external magnetic field and this gives the quantization condition of Dirac in geometric quantization. [6] Also the algebra of Hamiltonian vector fields forms an infinite dimensional symmetry group with a central extension.

In this letter, we explore the Hamiltonian formalism of nonabelian CSQM, i.e. isospin particle interacting with external Yang-Mills fields without kinetic or potential energy term. To describe the motion of isospin particle in external Yang-Mills fields, one needs to introduce isospin vector $Q^{a}(t)[7]$ which transforms under the adjoint representation of the internal symmetry group. We will consider only $S U(2)$ case. The dynamics is determined by the nonabelian generalizations of the Lorentz equations and the equations for $Q^{a}(t)$ for which there is no abelian counterpart. These equations are known as Wong's equations. Later, Lagrangian formul-tion of Wong's equations were given in fermionic language and bosonic language[8-10]. We follow ref. [10] for our purpose.

Consider the action[10] given by

$$
S=\int\left[i \operatorname{Tr}\left(K g^{-1} \dot{g}\right)-\dot{q}^{j} \operatorname{Tr}\left(g K g^{-1} A_{j}(q)\right)\right] d t
$$

Here $K^{\prime}=K^{a} \sigma^{a}, \sigma^{a}$ 's are Pauli matrices and $K^{a}$ are some real constants. $g$ is an element of $S U(2)$ and $A_{j} \equiv A_{j}^{a} \sigma^{a}$ is the external Yang-Mills field. We put gauge charge $e=1$. The above action is invariant under

- gauge transformations:

$$
g \rightarrow h g, \quad A_{i} \rightarrow h A_{i} h^{-1}+h \partial_{i} h^{-1}
$$


- time reparametrizations $t \rightarrow t^{\prime}(t)$.

It describes motion of isospin particle in $n$-dimensional space.$/$ in the presence of external Yang-Mills field without kinetic or potential energy term and it is n nonabelian generalization of Chern-Simons term.

Rewriting the above Lagrangian using $g=\exp \left(i \frac{\sigma^{a}}{2} \theta^{a}\right)$ anc defining the isospin charge by

$$
Q^{a}=-\operatorname{Tr}\left(K g^{-1} \sigma^{a} g\right)
$$

we have

$$
S=\int_{j}\left[\frac{1}{2} Q^{a} \dot{\theta}^{a}+A_{j}^{a}(q) Q^{a} \dot{q}^{j}\right] d t
$$

Using $\frac{\partial Q^{a}}{\partial \theta^{b}}=-\epsilon^{a b c} Q^{c}$ from Eq. (3), the Euler-Lagrange equations become

$$
\begin{aligned}
& F_{i j}^{a} Q^{a} \dot{q}^{j}=0 \\
& \dot{Q}^{a}=\epsilon^{a b c} A_{j}^{b} Q^{c} \dot{q}^{j}
\end{aligned}
$$

The above equations are kilown as Wong's equations[ $[\bar{l}$ without acceleration term. These equations require the velocity vector $\dot{q}^{j}$ to be a null eigenvector of $F_{i j} \equiv F_{i j}^{a} Q^{a}$. When the matrix $F_{i j}$ is non-degenerate, we have $\dot{q}^{j}=0$ and $Q^{a}=$ constant.

From Eq. (6), we see that $Q^{a} Q^{a}=$ constant. This describes the precessional motion of $Q^{u}$ 's. We introduce this as a constraint by including $Q^{a} Q^{a}=$ constant term in the Lagrangian and our final form of the action becomes

$$
S=\int\left[\frac{1}{2} Q^{a} \dot{j}^{a}+A_{j}^{a}(q) Q^{a} \dot{q}^{j}+\lambda\left(Q^{2}-m^{2}\right)\right]
$$

where $\lambda$ is some undetermined multiplier and $m$ is a constant.

To clescribe Hamiltonian formalism, we have to find all the constraints of our system and whether they are first-or second-class. The conjugate momenta to 0.q. and $\lambda$ are given from $\mathrm{Eq} .(\bar{T})$,

$$
\begin{aligned}
& p^{a}=\frac{\partial L}{\partial \dot{\partial} a}=\frac{1}{2} Q^{a} \\
& p_{i}=\frac{\partial L}{\partial \dot{q}^{i}}=A_{i}^{a} Q^{a} \\
& p_{\lambda}=\frac{\partial L}{\partial \dot{\lambda}}=0
\end{aligned}
$$


We choose $\lambda$ as a dynamical variable in order to apply Dirac procedure in a strict fashion. Since none of the conjugate momentum depend on the velocity: we have prirnary constraints

$$
\begin{aligned}
& \phi^{a}=p^{a}-\frac{1}{2} Q^{a} \approx 0 \\
& \dot{\phi}_{i}=p_{i}-A_{i}^{a} Q^{a} \approx 0 \\
& \Phi=p_{\lambda} \approx 0
\end{aligned}
$$

The canonical Hamiltonian is given by

$$
H_{c}=p^{a} \dot{\theta}^{a}+p_{i} \dot{q}^{i}+p_{\lambda} \dot{\lambda}-L=\dot{\lambda} p_{\lambda}-\lambda\left(Q^{2}-m^{2}\right)
$$

and we define the total Hamiltonian by

$$
H=v^{a} \phi^{a}+v^{i} \phi_{i}+\Lambda \Phi+\dot{\lambda} p_{\lambda}-\lambda\left(Q^{2}-m^{2}\right)
$$

where $v^{a}, v^{i}$, and $\Lambda$ are some undetermined Lagrange multipliers. The condition that the primary constraints must be obeyed for all time gives us

$$
\begin{aligned}
& \left.\dot{\phi}^{a}\right|_{P}=\left.\left\{\phi^{a}, H\right\}\right|_{P}=-\epsilon_{a b c} v^{b} Q^{c}-v^{i} \epsilon_{a b c} A_{i}^{b} Q^{c}=0 \\
& \left.\dot{\phi}_{i}\right|_{P}=\left.\left\{\phi_{i}, H\right\}\right|_{P}=-\epsilon_{a b c} A_{i}^{a} v^{b} Q^{c}+v^{j} A_{[i, j]}^{a} Q^{a}=0 \\
& \left.\dot{\Phi}\right|_{P}=\left.\{\Phi, H\}\right|_{P}=Q^{2}-m^{2}=0
\end{aligned}
$$

where $P$ denotes the subspace of $T^{*} M \times T^{*} G(G: S U(2)$ group manifold) defined by the constraint equations in $\mathrm{Eq}$. (9). In the above, we used fundamental commutation relations $\left\{p^{a}, \theta^{b}\right\}=-\delta_{a b},\left\{p_{i}, q_{j}\right\}=-\delta_{i j},\left\{p_{\lambda}, \lambda\right\}=-1$ and all others are zero. Also from $\frac{\partial Q^{a}}{\partial \theta^{b}}=-\epsilon_{a b c} Q^{c}$, we have

$$
\left\{p^{2}, Q^{b}\right\}=\epsilon^{a b c} Q^{c}
$$

From Eq. (12), we have

$$
v^{b}=V Q^{b}-v^{j} A_{j}^{b} \quad(V \text { : arbitrary })
$$

Substituting the above into Eq. (1:3). we have

$$
i^{j} F_{i,}^{d} Q^{a}=0
$$


We see from the above equation that if the rank of the matrix $F_{i j}$ is $r, r$ of $v^{j}$ 's or linear combinations of them are zero while the rest are arbitrary. A secondary constraint has been generated and is given by

$$
\tilde{\Phi}=Q^{2}-m^{2} \approx 0
$$

Adding the new constraint and substituting Eq. (16) into the total Hamiltonian in Eq.(11), we get a new Hamiltonian

$$
H^{\prime}=V \phi^{a} Q^{a}+v^{i}\left(p_{i}-A_{i}^{a} p^{a}-\frac{1}{2} A_{i}^{a} Q^{a}\right)+\tilde{\Lambda} \tilde{\Phi}+(\lambda-\operatorname{term})
$$

where $\tilde{\Lambda}$ is another undetermined Lagrangian multiplier. With $H^{\prime}$, we can check that no new constraints arise from requiring the preservation of constraints in time.

Now we separate first class constraints and second class constraints. First, we see that $\Phi \approx 0$ of Eq. (14) and $\tilde{\Phi} \approx 0$ of Eq.(18) are first class constraints since their bracket with all other constraint vanishes. We can choose the gauge $\lambda=\tilde{\Lambda}=0$ without changing the dynamics. And we will drop all reference to $\lambda, \tilde{\Lambda}$ and the conjugate momentum $p_{\lambda}$ from now on. Among $\phi^{a} \approx 0$, there are one first class constraint and two second class constraints. The first class constraint is given by

$$
\phi^{(1)}=Q^{a} \phi^{a} \approx 0
$$

It commutes with all other constraints

$$
\left\{\phi^{(1)}, \phi^{a}\right\}=\left\{\phi^{(1)}, \phi_{i}\right\}=0
$$

Using this, we fix the gauge $V=0$. For the remaining two second class constraints, we take the linear combination of $\phi^{a}$ 's:

$$
\begin{aligned}
& \phi^{\mu}=a_{a}^{\mu} \phi^{a} \\
& a_{a}^{\mu} a_{a}^{\nu}=\delta^{\mu \nu} \quad(\mu, \nu=1,2) \\
& m \epsilon^{\mu \nu} a_{a}^{\mu} a_{b}^{\nu}=\epsilon_{a b c} Q^{c}
\end{aligned}
$$

If the rank of $F_{i j}$ is $r$, there are $n-r$ first class constraints which are linear combinations of $\phi^{a}$ and $\phi_{i}$. Conicler

$$
\phi_{\alpha}=\Lambda_{\alpha \alpha}^{i}\left(o_{i}+. A_{i}^{a} \phi^{a}\right)
$$


We can easily check that

$$
\begin{aligned}
& \left\{\phi_{\alpha}, \phi^{a}\right\}=0 \\
& \left\{\phi_{\alpha}, \phi_{i}\right\}=\Lambda_{\alpha}^{j} F_{i j}^{a} Q^{a}=0
\end{aligned}
$$

if $\Lambda_{\alpha}^{i}(\alpha=1, \ldots, n-r)$ is a null eigenvector of the matrix $F_{i j}$ and $\phi_{\alpha}$ 's are first class constraints. We take the remaining $r$ second class constraints to be $\phi_{i}(i=1, \ldots, r)$.

In general, $\Lambda_{\alpha}^{i}$ could depend on $Q^{a}$ 's. However, we assume that $\Lambda_{\alpha}^{i}$ depend only on $q$ 's and not on $Q^{\prime \prime \prime}$ 's. We also assume that $r$ is constant at each $q$, i.e. $\operatorname{ker} F$ is a distribution of $(n-r)$ subspaces of $M$. Unlike the abelian case[1], $\operatorname{ker} F$ is in general not integrable. We will restrict ourselves to the case when $\operatorname{Ker} F$ is integrable, $M$ is foliated with $(n-r)$ dimensional leaves and $\operatorname{ker} F$ is tangent space to the leaf at each $q$. For example, we will consider later isospin particle in 't Hooft-Polyakov magnetic monopole field and this satisfies the above assumptions. We can choose local coordinate $\left\{q^{i}\right\}$ in some neighborhood of each point $q$ such that these coordinates are adapted to the foliation. We will work from now on with this coordinate system which is adapted to the foliation and for notational convenience, we will denote this coordinate system by the same symbol $q^{i}$ 's. In this coordinate system $q^{1}, \ldots, q^{r}$ parametrize the different leaves, and $q^{r+1}, \ldots, q^{n}$ are coordinates in the leaves.

In this coordinate system, we see from Eq. (17) that $v^{i}=0$ for $i=1, \ldots, r$ and $v^{i}=$ arbitrary for $i=r+1, \ldots, n$. We will use this arbitrariness to choose the gauge

$$
q^{i} \approx \text { constant }, \quad i=r+1, \ldots, n
$$

The preservation of this gauge condition in time requires $v^{i}=0$ for $i=r+1, \ldots, n$ as can be easily seen from Hamiltonian Eq. (19) with $\lambda=\dot{\Lambda}=p_{\lambda}=V=0$. The motion takes place entirely in the reduced phase space $\Gamma$ which is the $(r+2)$ dimensional subspace of $T^{*} M \times T^{*} G$ defined by the first two equations of Eqs.(9), (28) and (18). On $\Gamma$, we have solutions of Wong's Eqs.(5) and (6) such that $\dot{q}^{i}=0(i=1, \ldots, r)$, $Q^{a}=$ constant which satisfy Eq.(18) and the Hamiltonian (19) is zero. Other dynamical variables can be eliminated by the first two Eqq. of (9) and Eq.(28). Therefore the reduced phase space $\Gamma$ can be identified as $\Gamma_{r} \times S^{2}$, where $\Gamma_{r}$ is the $r$-dimensional 
subspace of $M$ defined by the foliation (27) and (2S) and $S^{2}$ is defined by (1S). $S^{2}$ is the coadjoint orbit of $S U(2)$ group[12].

We calculate the Dirac bracket on $\Gamma$. Denoting the constraints by $\phi_{A}=\left(\phi^{\mu}, \phi_{i}\right)$ $(\mu=1,2, i=1, \ldots, r)$ and the matrix $\Theta_{A B}=\left\{\phi_{A}, \phi_{B}\right\}$, we have

$$
\Theta_{A B}=\left(\begin{array}{cc}
-m \epsilon^{\mu \nu} & -a_{a}^{\mu} \epsilon_{a b c} A_{j}^{b} Q^{c} \\
a_{a}^{\nu} \epsilon_{a b c} A_{i}^{b} Q^{c} & A_{[i, j]}^{a} Q^{a}
\end{array}\right)
$$

The inverse matrix is given by

$$
\left(\Theta^{-1}\right)_{A B}=\left(\begin{array}{cc}
\frac{1}{m} \epsilon^{\mu \nu}+F_{i j}^{-1} a_{b}^{\mu} a_{c}^{\nu} A_{i}^{b} A_{j}^{c} & -a_{a}^{\mu} A_{i}^{a} F_{i j}^{-1} \\
a_{a}^{\nu} A_{j}^{a} F_{j i}^{-1} & F_{i j}^{-1}
\end{array}\right)
$$

In the above expression $F^{-1}$ is the inverse matrix of $F_{i j} \equiv F_{i j}^{a} Q^{a}$. The Dirac bracket is defined by

$$
\{\xi, \eta\}^{*}=\{\xi, \eta\}-\left\{\xi, \phi_{A}\right\}\left(\Theta^{-1}\right)_{A B}\left\{\phi_{B}, \eta\right\}
$$

A short calculation gives the following Dirac brackets among the dynamical variables

$$
\begin{aligned}
& \left\{Q^{a}, Q^{b}\right\}^{*}=\epsilon^{a b c} Q^{c} \\
& \left\{q^{i}, q^{j}\right\}^{*}=\left(F^{-1}\right)_{i j} \\
& \left\{Q^{a}, q^{i}\right\}^{*}=0
\end{aligned}
$$

where we used $Q^{2}=m^{2}$ in the first bracket.

We can formulate the theory directly on the reduced phase space $\Gamma$ by using the simplectic structure on $\Gamma$. We can parametrize $Q^{\prime} s$ on $S^{2}$ by

$$
\begin{aligned}
& Q^{1}=m \sin \theta \cos \phi \\
& Q^{2}=m \sin \theta \sin \phi \\
& Q^{3}=m \cos \theta
\end{aligned}
$$

where $0, \phi$ are the spherical coordinates on $S^{2}$. Consider one-form

$$
v=-m \cos \theta d \phi+c A_{i}^{a} Q^{a} d q^{i}
$$


and the topological action

$$
S=\int\left(-m \cos 0 \dot{\phi}+e A_{i}^{a} Q^{a} \dot{q}^{i}\right)
$$

In the above we restored gauge charge $e$ for later discussion. The Euler-Lagrange equations obtained from the above action become Eqs. (5) and (6). Define two-form $\Omega$ on $\Gamma$ by $\Omega=d v$. Then, we have

$$
\Omega=\omega+\mu
$$

where

$$
\begin{aligned}
& \omega=e F_{i j}^{a} Q^{a} d q^{i} \wedge d q^{j} \\
& \mu=m \sin \theta d \theta \wedge d \phi
\end{aligned}
$$

using $d Q^{a}=e \epsilon^{a b c} A_{i}^{b} d q^{i} Q^{c}$ from Eq. (6). $\Omega$ is closed and non-degenerate on $\Gamma$ and defines symplectic two-form on $\Gamma$. Let us define Poisson bracket on $\Gamma[11]$ by

$$
\{f, g\}=\left(F^{-1}\right)_{i j} \frac{\partial f}{\partial q^{i}} \frac{\partial g}{\partial q^{j}}+\frac{1}{m \sin \theta}\left\{\frac{\partial f}{\partial \phi} \frac{\partial g}{\partial \theta}-\frac{\partial f}{\partial \theta} \frac{\partial g}{\partial \phi}\right\}
$$

where $f$ and $g$ are arbitrary functions of $q^{i}$ and $\theta, \phi$ and $F^{-1}$ is the inverse matrix of $e F_{i j}^{a} Q^{a}$. We can easily check that the Dirac bracket relations in Eq. (32) can be obtained by using the above Poisson bracket and the expression for $Q^{a}$ 's up to trivial redefinitions of $Q^{\prime} s$. So the actions (7) and (37) describes the same dynamics on the reduced phase space $\Gamma$.

The canonical quantization can be obtained by replacing the Dirac bracket by $-i$ times the commutators. Here we discuss possible implication of symplectic structure on $\Gamma$ of isospin particle in 't Hooft-Polyakov magnetic monopole field in terms of geometric quantization[6]. In geometric quantization, the Hilbert space is constructed from the complex vector space of cross section of a $U(1)$ bundle $L$ over the symplectic manifold $(\Gamma, \Omega)$. Given any classical observable, we can define its Hamiltonian vector field and we can associate with it a differential operator which acts on the sections of $L$ by lifting the Hamiltonian vector fields using the connection on $L$. The curvature constructed from the connection must be proportional to the pull back of $\Omega$ in order 
to satisfy the quantum condition of Dirac. For the symplectic manifold $(\Gamma, \Omega)$ to be quantizable, $\Omega$ must define an integral cohomology class on $\Gamma$, i.e.

$$
\int_{N} \Omega=2 \pi k \quad(k \in Z)
$$

over any integer 2-cycle $N$ in $\mathrm{I}$. This is known as prequantization condition in geometric quantization.

Let us consider an example which describes previous results by considering isospin particle is 't Hooft-Polyakov magnetic monopole background [13]. The vector potential is given by the Wu-Yang ansatz

$$
A_{0}^{a}=n^{a} \frac{f(r)}{e r}, \quad A_{i}^{a}=\epsilon^{a i j} n^{j} \frac{1-h(r)}{e r}
$$

Here $n^{i} \equiv q^{i} / r$ is the unit vector. Using the above equation with $f(r)=0$, we find

$$
F_{i j}^{a}=-\frac{1}{e}\left[\frac{h^{2}-1}{r^{2}} \epsilon_{i j k} n^{k} n^{a}+\frac{h^{\prime}}{r}\left(\epsilon_{a i j}-\epsilon_{i j k} n^{k} n^{a}\right)\right]
$$

We consider the asymptotic region $r \rightarrow$ large where it is known that $h(r)$ falls off exponentially and we have

$$
F_{i j}^{a} Q^{a} \rightarrow \frac{1}{e r^{2}} \epsilon_{i j k} n^{k} n^{a} Q^{a}
$$

Substituting the above equation into $\mathrm{Eq}$. (17), we have

$$
\frac{1}{e r^{2}} \epsilon_{i j k} n^{k} n^{a} Q^{a} \Lambda_{\alpha}^{i}=0
$$

We can easily check that $\alpha=1$ and $\Lambda^{i}$ is given by

$$
\Lambda^{i} \sim n^{i}
$$

i.e. the leaves of the foliation $\operatorname{ker} F$ are one-dimensional and the direction is radial everywhere. $\Gamma_{r}$ is given by $S^{2}$ and the reduced phase is product of two $S^{2}$, one for space and the other for internal space.

Finally we discuss possible implication of prequantization condition of Eq. (42). Integrating over internal $S^{2}$, we have

$$
m=\frac{k}{2}
$$


and it gives the isospin quantization. Using the expression (45) and integrating over space $S^{2}$, we have $Q=k / 2\left(Q=n^{a} Q^{a}\right)$. So isospin quantization is consistent with the Schwinger condition $e g=1$, where $g$ is the magnetic charge. However, let us assume that there exist, in general, solutions of the form

$$
F_{i j}^{a} \rightarrow \frac{g}{r^{2}} \epsilon_{i j k} n^{k} n^{a}
$$

in the asymptotic region $r \rightarrow$ large. 't Hooft-Polyakov monopole solution( and PrasadSommerfeld solution) corresponds to $g=1 / e$ as in Eq.(45). For solutions of above form, we see that the quantization condition gives $e g=p / q(p, q$;integers $)$ and it suggests that product of gauge charge and magnetic charge can be fractional. Of course $p / q=1 / 2$ corresponds to Dirac condition. It would be intereting to see whether such solutions indeed exist.

\title{
Acknowledgements
}

I like to thank Dr. J. Hong for useful discussions. I also like to thank Prof. P. Ramond for reading the manuscript and encouragement. This work was supported by Korea Science and Engineering Foundation and in part by the Institute for Fundamental Theory at University of Florida.

\section{DISCLAIMER}

\begin{abstract}
This report was prepared as an account of work sponsored by an agency of the United States Government. Neither the United States Government nor any agency thereof, nor any of their employees, makes any warranty, express or implied, or assumes any legal liability or responsibility for the accuracy, completeness, or usefulness of any information, apparatus, product, or process disclosed, or represents that its use would not infringe privately owned rights. Reference herein to any specific commercial product, process, or service by trade name, trademark, manufacturer, or otherwise does not necessarily constitute or imply its endorsement, recommendation, or favoring by the United States Government or any agency thereof. The views and opinions of authors expressed herein do not necessarily state or reflect those of the United States Government or any agency thereof.
\end{abstract}




\section{References}

[1] R. Floreanini, R. Fercacci, and E. Sezgin, Nucl. Phys. B 322 (1989) 255.

[2] P. S. Howe and P. K. Townsend, Class. Quant. Grav. 7 (1990) 427.

[3] E. Witten, Comm. Math. Phys. 121 (1989) 351.

[4] G. V. Dunne, R. Jackiw and C. A. Trugenberger, Phys. Rev. D 41 (1990) 661; G. Papadopoulos, Phys. Lett. B 248 (1990) 113; T. Matsuyama, J. Phys. A 23 (1990) 5241; M. Blau, Preprint CPT-90/P.2369 (1990); R. Reuter, Phys. Rev. D $42(1990) 2763$.

[5] P. A. M. Dirac, Lectures on Quantum Mechanics (Yeshiva Univ., New York, 1964); E. C. G. Sudarshan and N. Mukunda, Classical Dynamics, a Modern Perspective (Wiley, New York, 1974).

[6] N. Woodhouse, Geometric Quantization (Clarendon Press, Oxford ,1980).

[7] S. K. Wong, Nuovo Cim. A 65 (1970) 689.

[8] A. P. Balachandran, P. Salomonson, and B.-S. Skagerstam and J.-O. Winnber, Phys. Rev. D 15 (1977) 2308.

[9] A. Barducci, R. Casalbuoni and L. Lusanna, Nucl. Phys. B 124 (1977) 93.

[10] A. P. Balachandran, S. Borchardt and A. Stern, Phys. Rev. D 17 (1978) 3247.

[11] F. A. Berezin, Commun.Math.Phys. 40 (1975) 153.

[12] See, for example, C. Duval and P. Horvathy, Ann. Phys. 142 (1982) 10.

[13] For a review see A. Actor, Rev. Mod. Phys. 51 (1979) 461. 
s.
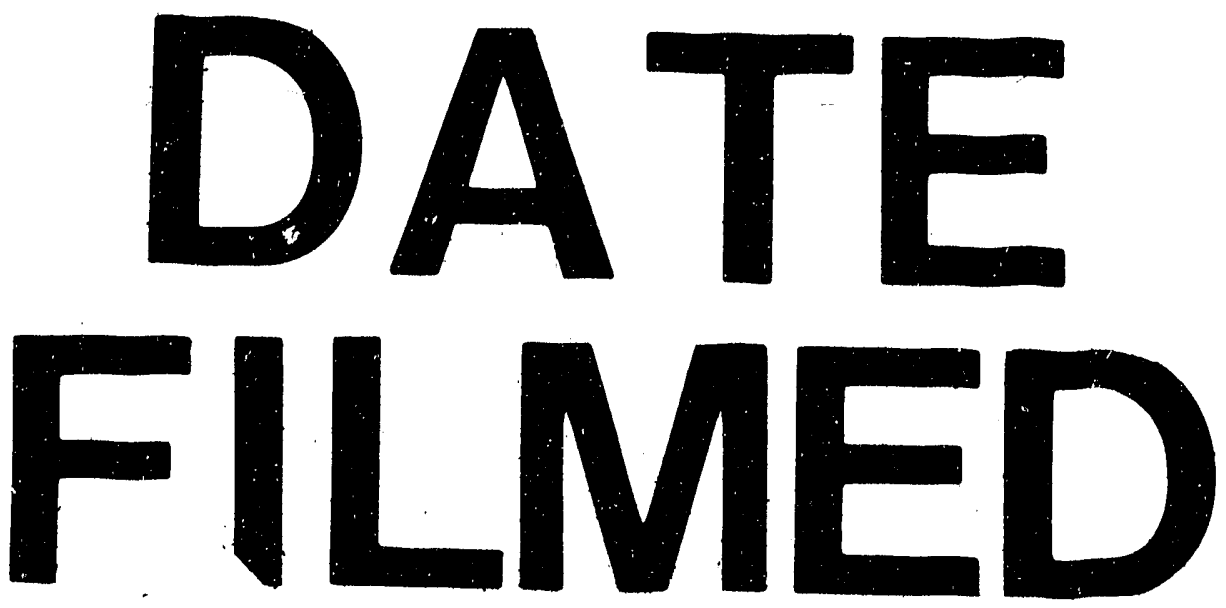

$8 / 14 / 92$ 